\title{
On the Existence and Stability of Periodic Solutions for a Nonlinear Neutral Functional Differential Equation
}

\author{
Yueding Yuan ${ }^{1,2,3}$ and Zhiming Guo ${ }^{1,3}$ \\ ${ }^{1}$ School of Mathematics and Information Sciences, Guangzhou University, Guangzhou 510006, China \\ ${ }^{2}$ School of Mathematics and Computer Sciences, Yichun University, Yichun 336000, China \\ ${ }^{3}$ Key Laboratory of Mathematics and Interdisciplinary Science of Guangdong Higher Education Institutes, \\ Guangzhou University, Guangzhou 510006, China
}

Correspondence should be addressed to Zhiming Guo; gzm100@21cn.com

Received 31 January 2013; Accepted 1 March 2013

Academic Editor: Chuangxia Huang

Copyright (C) 2013 Y. Yuan and Z. Guo. This is an open access article distributed under the Creative Commons Attribution License, which permits unrestricted use, distribution, and reproduction in any medium, provided the original work is properly cited.

This paper deals with the existence and stability of periodic solutions for the following nonlinear neutral functional differential equation $(d / d t) D u_{t}=p(t)-a u(t)-a q u(t-r)-h(u(t), u(t-r))$. By using Schauder-fixed-point theorem and Krasnoselskii-fixedpoint theorem, some sufficient conditions are obtained for the existence of asymptotic periodic solutions. Main results in this paper extend the related results due to Ding (2010) and Lopes (1976).

\section{Introduction}

In recent years, the existence and stability of periodic solutions for differential equation has been extensively studied. Many researchers used the Lyapunov functional method, Hopf bifurcation techniques, and Mawhin continuation theorems to obtain the existence and stability of periodic solutions for neutral functional differential equation (NFDE); see papers [1-14] and their references therein. However, researches on the existence and stability of periodic solutions for NFDE by using fixed-point theorem are relatively rare $[15,16]$. The reason lies in the fact that it is difficult to construct an appropriate completely continuous operator and an appropriate bounded closed convex set.

In this paper, we will investigate the existence and stability of periodic solutions for the following nonlinear NFDE

$$
\frac{d}{d t} D u_{t}=p(t)-a u(t)-a q u(t-r)-h(u(t), u(t-r)),
$$

where $D u_{t}=u(t)-q u(t-r),|q|\langle 1, a\rangle 0, h \in C(\mathbb{R} \times \mathbb{R}, \mathbb{R})$, and $p \in C(\mathbb{R}, \mathbb{R})$. Such a kind of NFDE has been used for the study of distributed networks containing a transmission line $[17,18]$. For example, suppose a system consists of a long electrical cable of length $l$, and one end of which isconnected to a power source $E(t)$ with resistance $R_{0}$, while the other end is connected to an oscillating circuit formed of a condenser $C_{0}$ and a nonlinear element, the volt-ampere characteristic of which is $i=f(u)$. Let $L, C, R$, and $G$ be the parameters of the transmission line, respectively, $Z_{0}$ the characteristic impedance of the line, $v=1 / \sqrt{L C}$ the propagation velocity and assume the losses can not be disregarded. The process of the final end volt $u(t)$ in such a system can be described by the following NFDES:

$$
\begin{aligned}
u^{\prime}(t)-q u^{\prime}(t-r)= & p(t)-a u(t)-a q u(t-r) \\
& -b f(u(t))+b q f(u(t-r))
\end{aligned}
$$

or

$$
\begin{aligned}
C_{0}\left(u^{\prime}(t)-k u^{\prime}(t-r)\right)= & p(t)-Z u(t)-Z k u(t-r) \\
& -f(u(t)-k u(t-r)),
\end{aligned}
$$

where $a=1 / Z_{0} C_{0}, b=1 / C_{0}, q=\left(Z_{0}-R_{0}\right) / A^{2}\left(Z_{0}+R_{0}\right)$, $k=\left(1-Z R_{0}\right) /\left(1+Z R_{0}\right), p(t)=2 E(t-(r / 2)) / A\left(Z_{0}+R_{0}\right) C_{0}$, $r=2 l / v, A=e^{R l / Z_{0}}, Z=\sqrt{C / L}$, and $f(u)$ is a given nonlinear function. If $R_{0}>0$, then $|q|<1,|k|<1$. Obviously, we see that (2) (or (3)) is a special case of (1). The aim of this paper 
is to establish some criteria to guarantee the existence and stability of periodic solution for (1) by using Schauder's fixedpoint theorem and Krasnoselskii's fixed-point theorem. The interesting is that main results obtained in this paper extend the related existing results. Furthermore, our results can also be applied to solve the problem of the existence and stability of periodic solutions for (2) and (3).

\section{Main Results and Proofs}

In this section, let $C^{1}\left(\mathbb{R}^{N}\right)\left(C\left(\mathbb{R}^{N}\right)\right)$ denote the set of all continuously differentiable functions (all continuous functions) $\phi: \mathbb{R}^{N} \rightarrow \mathbb{R}$, where $N=1,2 . C_{\omega}=\{\phi \mid \phi \in C(\mathbb{R}), \phi(t+$ $\omega)=\phi(t)\}$ is a Banach space with the supremum norm $\|\cdot\|_{0}$, $C_{\omega}^{1}=C^{1}(\mathbb{R}) \cap C_{\omega}$ with the norm $\|\phi\|_{1}=\|\phi\|_{0}+\left\|\phi^{\prime}\right\|_{0}$ in a period interval, and $\omega$ is a positive constant. The next lemma will be used in the sequel.

Lemma 1. If $a \neq 0, f \in C_{\omega}$, then the scalar equation $x^{\prime}(t)=$ ax $(t)+f(t)$ has a unique $\omega$-periodic solution:

$$
x(t)=\left(1-e^{a \omega}\right)^{-1} \int_{t}^{t+\omega} e^{a(t+\omega-s)} f(s) d s .
$$

Proof. It is easy to prove. We can find it in many ODE textbooks (e.g., see Example 2 on page 35 of [19]).

Theorem 2. Suppose that $h \in C\left(\mathbb{R}^{2}\right)$ and $p \in C_{T}$. If there exists a constant $H>0$ such that

$$
\|p\|_{0}<(1-3|q|) a H-\sup _{|x|,|y| \leq H}|h(x, y)|
$$

then (1) has a T-periodic solution.

Proof. According to the condition (5), we can find a sufficiently small $L>0$ such that

$$
\left(2 L+\frac{1}{a}\right)\left[\|p\|_{0}+2 a|q| H+\sup _{|x|,|y| \leq H}|h(x, y)|\right]+|q| H \leq H .
$$

Let $v(t)=u(L t), \tau=r / L, p_{1}(t)=p(L t)$, and $\omega=T / L$; then (1) can be rewritten as

$$
\begin{aligned}
v^{\prime}(t)-q v^{\prime}(t-\tau)= & L p_{1}(t)-a L v(t)-a q L v(t-\tau) \\
& -\operatorname{Lh}(v(t), v(t-\tau)),
\end{aligned}
$$

where $p_{1}(t) \in C_{\omega}$ with $\|p\|_{0}=\left\|p_{1}\right\|_{0}$. It suffices to prove that (7) has a $\omega$-periodic solution. Let

$$
M=\left\{\phi \mid \phi \in C_{\omega}^{1},\|\phi\|_{1} \leq H\right\} .
$$

Then $M$ is a bounded closed convex set of the Banach space $C^{1}(\mathbb{R})$. For any given $\phi \in M$, consider the nonhomogeneous equation:

$$
\begin{aligned}
v^{\prime}(t)= & -a L v(t)+L p_{1}(t)-a q L \phi(t-\tau) \\
& -\operatorname{Lh}(\phi(t), \phi(t-\tau))+q \phi^{\prime}(t-\tau) .
\end{aligned}
$$

According to Lemma 1, (9) has a unique $\omega$-periodic solution:

$$
\begin{aligned}
v(t)=\left(1-e^{-a L \omega}\right)^{-1} \\
\times \int_{t}^{t+\omega} e^{-a L(t+\omega-s)} \\
\quad \times\left[L p_{1}(s)-a q L \phi(s-\tau)\right. \\
\left.\quad-\operatorname{Lh}(\phi(s), \phi(s-\tau))+q \phi^{\prime}(s-\tau)\right] d s .
\end{aligned}
$$

Define an operator $A$ by

$$
\begin{aligned}
& (A \phi)(t) \\
& =\left(1-e^{-a L \omega}\right)^{-1} \\
& \quad \times \int_{t}^{t+\omega} e^{-a L(t+\omega-s)} \\
& \quad \times\left[L p_{1}(s)-a q L \phi(s-\tau)\right. \\
& \left.\quad-L h(\phi(s), \phi(s-\tau))+q \phi^{\prime}(s-\tau)\right] d s \\
& \quad \times \int_{t}^{t+\omega} e^{-a L(t+\omega-s)} \\
& \quad \times\left[L p_{1}(s)-2 a q L \phi(s-\tau)\right. \\
& \quad-L h(\phi(s), \phi(s-\tau))] d s+q \phi(t-\tau) .
\end{aligned}
$$

In order to prove that (7) has a periodic solution, we shall make sure that $A$ satisfies the conditions of Schauder's fixedpoint theorem (see Lemma 2.2.4 on page 40 of [20]).

Note that for any $x \in M$, we have $x(t+\omega)=x(t)$ and $\|x\|_{1} \leq H$

$$
\begin{aligned}
& (A x)(t+\omega) \\
& =\left(1-e^{-a L \omega}\right)^{-1} \\
& \quad \times \int_{t+\omega}^{t+2 \omega} e^{-a L(t+2 \omega-s)} \\
& \quad \times\left[L p_{1}(s)-2 a q L x(s-\tau)\right. \\
& \quad-L h(x(s), x(s-\tau))] d s+q x(t+\omega-\tau) \\
& =\left(1-e^{-a L \omega}\right)^{-1} \\
& \times \int_{t}^{t+\omega} e^{-a L(t+\omega-s)} \\
& \quad \times\left[L p_{1}(s)-2 a q L x(s-\tau)\right. \\
& \quad-\operatorname{Lh}(x(s), x(s-\tau))] d s+q x(t-\tau) \\
& =(A x)(t) ;
\end{aligned}
$$


Therefore, $(A x)(t+\omega)=(A x)(t)$. Meanwhile, we get

$$
\begin{aligned}
& (A x)^{\prime}(t) \\
& =\left(1-e^{-a L \omega}\right)^{-1} \\
& \times\left\{\int_{t}^{t+\omega} e^{-a L(t+\omega-s)}(-a L)\right. \\
& \times\left[L p_{1}(s)-2 a q L x(s-\tau)\right. \\
& -\operatorname{Lh}(x(s), x(s-\tau))] d s \\
& +\left(1-e^{-a L \omega}\right)\left[L p_{1}(t)-2 a q L x(t-\tau)\right. \\
& -\operatorname{Lh}(x(t), x(t-\tau))]\} \\
& +q x^{\prime}(t-\tau) \\
& =\left(1-e^{-a L \omega}\right)^{-1}(-a L) \\
& \times \int_{t}^{t+\omega} e^{-a L(t+\omega-s)} \\
& \times\left[\operatorname{Lp} p_{1}(s)-2 a q L x(s-\tau)\right. \\
& -\operatorname{Lh}(x(s), x(s-\tau))] d s \\
& +\left[\operatorname{Lp} p_{1}(t)-2 a q L x(t-\tau)-\operatorname{Lh}(x(t), x(t-\tau))\right] \\
& +q x^{\prime}(t-\tau) \text {. }
\end{aligned}
$$

By (6), we have

\section{$\|A x\|_{1}$}

$$
\begin{gathered}
=\|A x\|_{0}+\left\|(A x)^{\prime}\right\|_{0} \\
\leq \sup _{t \in \mathbb{R}} \mid\left(1-e^{-a L \omega}\right)^{-1} \\
\times \int_{t}^{t+\omega} e^{-a L(t+\omega-s)} \\
\times\left[\operatorname{Lp}_{1}(s)-2 a q L x(s-\tau)\right. \\
+\sup _{t \in \mathbb{R}} \mid\left(1-e^{-a L \omega}\right)^{-1}(-a L) \\
\times \int_{t}^{t+\omega} e^{-a L(t+\omega-s)} \\
\times[\operatorname{Lp}(x), x(s-\tau))] d s \mid \\
\quad-\operatorname{Lh}(x(s), x(s-\tau))] d s
\end{gathered}
$$

$$
\begin{aligned}
& +\left[L p_{1}(t)-2 a q L x(t-\tau)-L h(x(t), x(t-\tau))\right] \mid \\
& +|q|\|x\|_{1} \leq\left(2+\frac{1}{a L}\right) \\
& \times\left[L\left\|p_{1}\right\|_{0}+2 a|q| L H+L \sup _{|x|,|y| \leq H}|h(x, y)|\right] \\
& +|q| H=\left(2 L+\frac{1}{a}\right) \quad \\
& \quad \times\left[\left\|p_{1}\right\|_{0}+2 a|q| H+\sup _{|x|,|y| \leq H}|h(x, y)|\right]+|q| H \leq H .
\end{aligned}
$$

Thus, $A x \in M$.

For any $x \in M,\|A x\|_{0} \leq H,\left\|(A x)^{\prime}\right\|_{0} \leq H$. According to Arzela-Ascoli Theorem (see Theorem 4.9 .6 on page 84 of [21]), the subset $A M$ of $C_{\omega}$ is a precompact set; therefore, $A$ : $M \subset C^{1}(\mathbb{R}) \rightarrow C_{\omega}$ is a compact operator.

Suppose that $\left\{x_{n}\right\} \in M, x_{n} \rightarrow x$, then $\left\|x_{n}-x\right\|_{0} \rightarrow 0$ and $\left\|x_{n}^{\prime}-x^{\prime}\right\|_{0} \rightarrow 0$ as $n \rightarrow \infty$. Also, we have

$$
\begin{aligned}
& \left\|A x_{n}-A x\right\|_{0} \\
& =\sup _{t \in \mathbb{R}} \mid\left(1-e^{-a L \omega}\right)^{-1} \\
& \times \int_{t}^{t+\omega} e^{-a L(t+\omega-s)} \\
& \times\left[-2 a q L\left(x_{n}(s-\tau)-x(s-\tau)\right)\right. \\
& -L\left(h\left(x_{n}(s), x_{n}(s-\tau)\right)\right. \\
& -h(x(s), x(s-\tau)))] d s \\
& +q\left(x_{n}(t-\tau)-x(t-\tau)\right) \\
& \leq \frac{1}{a L}\left[2 a|q| L\left\|x_{n}-x\right\|_{0}\right. \\
& +L \sup _{t \in[0, \omega]} \mid h\left(x_{n}(t), x_{n}(t-\tau)\right) \\
& -h(x(t), x(t-\tau)) \mid] \\
& +|q|\left\|x_{n}-x\right\|_{0} \\
& =3|q|\left\|x_{n}-x\right\|_{0}+\frac{1}{a} \sup _{t \in[0, \omega]} \\
& \times\left|h\left(x_{n}(t), x_{n}(t-\tau)\right)-h(x(t), x(t-\tau))\right|, \\
& \left\|\left(A x_{n}\right)^{\prime}-(A x)^{\prime}\right\|_{0} \\
& =\sup _{t \in \mathbb{R}} \mid\left(1-e^{-a L \omega}\right)^{-1}(-a L)
\end{aligned}
$$




$$
\begin{aligned}
& \times \int_{t}^{t+\omega} e^{-a L(t+\omega-s)} \\
& \times[-2 a q L \\
& \times\left(x_{n}(s-\tau)-x(s-\tau)\right) \\
& -L\left(h\left(x_{n}(s), x_{n}(s-\tau)\right)\right. \\
& -h(x(s), x(s-\tau)))] d s \\
& -2 a q L\left(x_{n}(t-\tau)-x(t-\tau)\right)-L\left(h\left(x_{n}(t), x_{n}(t-\tau)\right)\right. \\
& -h(x(t), x(t-\tau)))+q\left(x_{n}^{\prime}(t-\tau)-x^{\prime}(t-\tau)\right) \\
& \leq 2 L\left[2 a|q|\left\|x_{n}-x\right\|_{0}\right. \\
& +\sup _{t \in[0, \omega]} \mid h\left(x_{n}(t), x_{n}(t-\tau)\right) \\
& -h(x(t), x(t-\tau)) \mid]+|q|\left\|x_{n}^{\prime}-x^{\prime}\right\|_{0} .
\end{aligned}
$$

When $\left\|x_{n}-x\right\|_{1} \rightarrow 0$ as $n \rightarrow \infty,\left|x_{n}(t)-x(t)\right| \rightarrow 0$ for $t \in[0, \omega]$ uniformly. And since $h$ is continuous, $\| A x_{n}-$ $A x\left\|_{0} \rightarrow 0,\right\|\left(A x_{n}\right)^{\prime}-(A x)^{\prime} \|_{0} \rightarrow 0$. Consequently, $A$ is continuous.

Thus, by Schauder-fixed-point theorem (see Lemma 2.2.4 on page 40 of [20]), there is a $\phi \in M$ such that $\phi=A \phi$. Therefore, (7) has at least one $\omega$-periodic solution. Since $v(t)=u(L t)$ and $p(L t)=p_{1}(t),(1)$ has at least one $T$-periodic solution. The proof is completed.

Next, we explore the stability of this $T$-periodic solution $u^{*}(t)$ for (1). We assume that theconditions of Theorem 2 are satisfied. Therefore, (1) has at least one $T$-periodic solution $u^{*}(t)$. Let $v(t)=u(t)-u^{*}(t)$ then (1) is transformed as

$$
\frac{d}{d t} D v_{t}=-a v(t)-a q v(t-r)-g(v(t), v(t-r)),
$$

where $D v_{t}=v(t)-q v(t-r)$ and $g(v(t), v(t-r))=h\left(u^{*}(t)+\right.$ $\left.v(t), u^{*}(t-r)+v(t-r)\right)-h\left(u^{*}(t), u^{*}(t-r)\right)$. Clearly, (16) has trivial solution $v(t) \equiv 0$. Now we use Krasnoselskiis-fixedpoint theorem (see [22] or [15, Lemma 2.2]) to prove that trivial solution $v(t) \equiv 0$ to (16) is asymptotically stable.

Set $S$ as the Banach space of bounded continuous function $\phi:[-r, \infty) \rightarrow R$ with the supremum norm $\|\cdot\|$. Also, Given the initial function $\psi$, denote the norm of $\psi$ by $\|\psi\|=$ $\sup _{t \in[-r, 0]}|\psi(t)|$, which should not cause confusion with the same symbol for the norm in $S$.

Theorem 3. Let $H$ be as in Theorem 2. Assume that all conditions of Theorem 2 are satisfied. Suppose that $h$ satisfies the Lipschitz condition and

$$
\sup _{|x|,|y| \leq H}|h(x, y)| \leq a H(1+|q|) .
$$

If there exists $Q>0$ such that

$$
\|\psi\| \leq \frac{Q-3|q| Q}{1+|q|}-H-\frac{1}{a(1+|q|)} \sup _{|x|,|y| \leq H+Q}|h(x, y)|,
$$

then the solution $v(\psi)(t)$ to (16) through $\psi$ satisfies $\lim _{t \rightarrow \infty} v(\psi)(t)=0$.

Proof. By (18), we have

$$
\begin{aligned}
& (1+|q|)\|\psi\|+3|q| \mathrm{Q}+\frac{1}{a} \sup _{|x|,|y| \leq H+\mathrm{Q}}|h(x, y)| \\
& +H(1+|q|) \leq \mathrm{Q} .
\end{aligned}
$$

Given the initial function $\psi$, by [20, Theorem 12.2.3], there exists a unique solution $v(\psi)(t)$ for (16). Let

$$
\begin{aligned}
& M_{\psi} \\
& \quad=\left\{\phi \mid \phi \in S,\|\phi\| \leq Q, \phi_{0}=\psi, \phi(t) \longrightarrow 0 \text { as } t \longrightarrow \infty\right\} ;
\end{aligned}
$$

then $M_{\psi}$ is a bounded convex closed set of $S$. We write (16) as

$$
\begin{aligned}
{[v(t)-q v(t-r)]^{\prime}=} & -a[v(t)-q v(t-r)]-2 a q v(t-r) \\
& -g(v(t), v(t-r))
\end{aligned}
$$

then we have

$$
\begin{aligned}
v(t)= & {[\psi(0)-q \psi(-r)] e^{-a t}+q v(t-r) } \\
& +\int_{0}^{t}[-2 a q v(s-r)-g(v(s), v(s-r))] e^{-a(t-s)} d s .
\end{aligned}
$$

For all $\phi \in M_{\psi}$, define the operators $A$ and $B$ by

$(A \phi)(t)$

$$
=\left\{\begin{array}{lr}
0, & t \in[-r, 0], \\
\int_{0}^{t}[-2 a q \phi(s-r)-g(\phi(s), \phi(s-r))] e^{-a(t-s)} d s, & t \geq 0,
\end{array}\right.
$$

$(B \phi)(t)$

$$
= \begin{cases}\psi(t), & t \in[-r, 0], \\ (\psi(0)-q \psi(-r)) e^{-a t}+q \phi(t-r), & t \geq 0 .\end{cases}
$$


For any $x, y \in M_{\psi}, x(t) \rightarrow 0, y(t) \rightarrow 0$ as $t \rightarrow \infty$, and $\|x\| \leq Q,\|y\| \leq Q$. Therefore, we have

$$
\begin{gathered}
\lim _{t \rightarrow \infty}(A x)(t) \\
=\lim _{t \rightarrow \infty} \frac{\int_{0}^{t}[-2 a q x(s-r)-g(x(s), x(s-r))] e^{a s} d s}{e^{a t}} \\
=\lim _{t \rightarrow \infty} \frac{1}{a}[-2 a q x(t-r)-g(x(t), x(t-r))]=0, \\
\lim _{t \rightarrow \infty}(B y)(t)=\lim _{t \rightarrow \infty}\left[(\psi(0)-q \psi(-r)) e^{-a t}+q y(t-r)\right]=0 .
\end{gathered}
$$

Thus, $\lim _{t \rightarrow \infty}(A x+B y)(t)=0$. Again by (17) and (19), we have

$$
\begin{aligned}
\| A x & +B y \| \\
\leq & \|A x\|+\|B y\| \\
= & \sup _{t \geq 0}\left|\int_{0}^{t}[-2 a q x(s-r)-g(x(s), x(s-r))] e^{-a(t-s)} d s\right| \\
& +\sup _{t \geq-r}|(B y)(t)| \\
\leq & {\left[2 a|q| Q+\sup _{|x|,|y| \leq H+Q}|h(x, y)|+\sup _{|x|,|y| \leq H}|h(x, y)|\right] } \\
& \times \sup _{t \geq 0}\left|\int_{0}^{t} e^{-a(t-s)} d s\right| \\
& +\max \left\{\|\psi\|, \sup _{t \geq 0}\left|(\psi(0)-q \psi(-r)) e^{-a t}+q y(t-r)\right|\right\} \\
& +\frac{1}{a} \sup _{|x|,|y| \leq H}|h(x, y)| \\
\leq & (1+|q|)\|\psi\|+3|q| Q+\frac{1}{a} \sup _{|x|,|y| \leq H+Q}|h(x, y)|
\end{aligned}
$$

Since $\left|(A x)^{\prime}(t)\right|=0, t \in[-r, 0]$, and

$$
\begin{aligned}
\left|(A x)^{\prime}(t)\right| & \mid-a \int_{0}^{t}[-2 a q x(s-r)-g(x(s), x(s-r))] e^{-a(t-s)} d s \\
= & -2 a q x(t-r)-g(x(t), x(t-r)) \mid \\
\leq & a\left[2 a|q| Q+\sup _{|x|,|y| \leq H+Q}|h(x, y)|+\sup _{|x|,|y| \leq H}|h(x, y)|\right] \\
& \times \sup _{t \geq 0}\left|\int_{0}^{t} e^{-a(t-s)} d s\right| \\
+ & 2 a|q| Q+\sup _{|x|,|y| \leq H+Q}|h(x, y)|+\sup _{|x|,|y| \leq H}|h(x, y)| \\
= & {\left[2 a|q| Q+\sup _{|x|,|y| \leq H+Q}|h(x, y)|+\sup _{|x|,|y| \leq H}|h(x, y)|\right], }
\end{aligned}
$$

here, the derivative of $(A x)^{\prime}(t)$ at zero means the left-hand side derivative when $t \leq 0$ and the right-hand side derivative when $t \geq 0$, one can see $(A x)^{\prime}(t)$ is bounded for all $x \in M_{\psi}$. Therefore, $A M_{\psi}$ is a precompact set of $S$. Thus, $A$ is compact.

Suppose that $\left\{x_{n}\right\} \subset M_{\psi}, x \in S, x_{n} \rightarrow x$ as $n \rightarrow \infty$; then $\left|x_{n}(t)-x(t)\right| \rightarrow 0$ uniformly for $t \geq-r$ as $n \rightarrow \infty$. Since

$$
\begin{aligned}
& \left\|A x_{n}-A x\right\| \\
& =\sup _{t \geq 0} \mid \int_{0}^{t}\left\{-2 a q\left[x_{n}(s-r)-x(s-r)\right]\right. \\
& -g\left(x_{n}(s), x_{n}(s-r)\right) \\
& +g(x(s), x(s-r))\} e^{-a(t-s)} d s \\
& \leq\left[2 a|q|\left\|x_{n}-x\right\|\right. \\
& +\sup _{t \geq 0} \mid h\left(u^{*}(t)+x_{n}(t), u^{*}(t-r)+x_{n}(t-r)\right) \\
& \left.-h\left(u^{*}(t)+x(t), u^{*}(t-r)+x(t-r)\right) \mid\right] \\
& \times \sup _{t \geq 0}\left|\int_{0}^{t} e^{-a(t-s)} d s\right|=2|q|\left\|x_{n}-x\right\| \\
& +\frac{1}{a} \sup _{t \geq 0} \mid h\left(u^{*}(t)+x_{n}(t), u^{*}(t-r)+x_{n}(t-r)\right) \\
& -h\left(u^{*}(t)+x(t), u^{*}(t-r)+x(t-r)\right) \mid \text {, }
\end{aligned}
$$


and $h$ is continuous, we have $\left\|A x_{n}-A x\right\| \rightarrow 0$ as $n \rightarrow \infty$. Thus, $A$ is continuous. Due to the fact that

$$
\begin{array}{r}
\|B x-B y\|=\sup _{t \geq 0}|q x(t-r)-q y(t-r)| \leq|q|\|x-y\|, \\
\forall x, y \in M_{\psi},
\end{array}
$$

and $|q|<1$, we know that $B$ is a contractive operator.

According to Krasnoselskii's fixed-point theorem (see [22] or [15, Lemma 2.2]), there is a $\phi \in M_{\psi}$ such that $(A+$ $B) \phi=\phi$. Therefore, $\phi(t)$ is a solution for (16). Because the solution through $\psi$ for the equation is unique, the solution $v(\psi)(t)=\phi(t) \rightarrow 0$ as $t \rightarrow \infty$.

When $h$ satisfies the Lipschitz condition, then there is a constant $L>0$ such that

$$
\begin{aligned}
& \left|h\left(u(t)+u^{*}(t), u(t-r)+u^{*}(t-r)\right)-h\left(u^{*}(t), u^{*}(t-r)\right)\right| \\
& \quad \leq L \sqrt{|u(t)|^{2}+|u(t-r)|^{2}}, \quad \forall u \in S .
\end{aligned}
$$

Since $\phi$ satisfies

$$
\begin{aligned}
\phi(t)= & {[\psi(0)-q \psi(-r)] e^{-a t}+q \phi(t-r) } \\
& +\int_{0}^{t}[-2 a q \phi(s-r)-g(\phi(s), \phi(s-r))] e^{-a(t-s)} d s,
\end{aligned}
$$

then

$$
\|\phi\| \leq(1+|q|)\|\psi\|+3|q|\|\phi\|+\frac{\sqrt{2} L}{a}\|\phi\|,
$$

that is

$$
\left(1-3|q|-\frac{\sqrt{2} L}{a}\right)\|\phi\| \leq(1+|q|)\|\psi\| .
$$

Therefore, if $1-3|q|-(\sqrt{2 L} / a)>0$, then there clearly exists a $\delta>0$ for any $\varepsilon>0$ such that $|\phi(t)|<\varepsilon$ for all $t \geq-r$ if $\|\psi\|<\delta$. Thus, we have the following.

Theorem 4. If the Lipschitz constant $L$ for $h$ corresponding to $\mathbb{R}^{2}$ satisfies

$$
1-3|q|-\frac{\sqrt{2} L}{a}>0,
$$

then the zero solution for (16) is stable.

When $p$ is constant and the equation $p-a(1+q) u=$ $h(u, u)$ has only one solution $u^{*}$, then $u^{*}$ is an equilibrium of (1) and (1) can be transformed to the following equation:

$$
\frac{d}{d t} D u_{t}=-a u(t)-a q u(t-r)-g(u(t), u(t-r)),
$$

where $D u_{t}=u(t)-q u(t-r)$ and $g(u(t), u(t-r))=h\left(u^{*}+\right.$ $\left.u(t), u^{*}+u(t-r)\right)-h\left(u^{*}, u^{*}\right)$. Now, we consider the stability of the zero solution for (34).
Theorem 5. Suppose that is $h \in C^{1}\left(\mathbb{R}^{2}\right)$ and $\left(h_{x}\left(u^{*}, u^{*}\right)\right.$, $\left.h_{y}\left(u^{*}, u^{*}\right)\right)=(0,0)$; then the zero solution of $(34)$ is exponentially asymptotically stable.

Proof. For all $\phi$ in $C=C([-r, 0], \mathbb{R})$, let

$$
\begin{gathered}
D \phi=\phi(0)-q \phi(-r), \\
L \phi=-a \phi(0)-a q \phi(-r), \\
F \phi=-g(\phi(0), \phi(-r)) .
\end{gathered}
$$

Then $D$ is stable, and $D$ and $L$ are linear and continuous. Consider the equation $(d / d t) D u_{t}=L u_{t}$. Let

$$
V(\phi)=(D \phi)^{2}+2 a q^{2} \int_{-r}^{0} \phi^{2}(\theta) d \theta .
$$

Then

$$
\begin{aligned}
\dot{V} & (\phi) \\
& =2(D \phi)(-a \phi(0)-a q \phi(-r))+2 a q^{2}\left(\phi^{2}(0)-\phi^{2}(-r)\right) \\
& =-2 a\left(1-q^{2}\right) \phi^{2}(0) .
\end{aligned}
$$

Thus, according to the last conclusion of Theorem 12.7.1 in [20, Page 297], the zero solution of $u^{\prime}(t)-q u^{\prime}(t-r)=$ $-a u(t)-a q u(t-r)$ is uniformly asymptotically stable. On the other hand, one can see that

$$
F_{\phi} u=\left(-g_{x}(u(0), u(-r)),-g_{y}(u(0), u(-r))\right) .
$$

Thus, $F(0)=F_{\phi}(0)=0$. According to [20, Theorem 12.9.1], the zero solution of (34) is exponentially asymptotically stable.

\section{Examples}

In this section, we present two examples to illustrate the applicability of our main results.

Example 6 (Lopes et al. $[8,9,13,15,23])$. Consider the NFDE (2) which arises from a transmission line model, where $a>$ $0, b>0, r>0,|q|<1, p \in C(\mathbb{R})$, and $f$ is a given nonlinear function. Now, let $h(u(t), u(t-r))=b f(u(t))-b q f(u(t-r))$. It is not difficult to see that (2) is a special case of (1). Therefore, by Theorems $2-5$, we have the following.

Theorem 7. Suppose that $f \in C(\mathbb{R})$ and $p \in C_{T}$. If there exists a constant $H>0$ such that

$$
\|p\|_{0}<(1-3|q|) a H-b(1+|q|) \sup _{|x| \leq H}|f(x)|,
$$

then (2) has a T-periodic solution.

Remark 8. Theorem 7 implies that the conditions in [15]

$$
l<1, \quad|q|<\frac{1-l}{3+l},
$$

where $l=(b / a H) \sup _{|x| \leq H}|f(x)|$, are unnecessary for the existence of periodic solutions for (2). 
Theorem 9. Let $H$ be as in Theorem 7. Assume that all conditions of Theorem 7 are satisfied. If $f$ satisfies the Lipschitz condition, $(b / a H) \sup _{|x| \leq H}|f(x)| \leq 1$ and there exists $Q>0$ such that

$$
\left\|\psi-u^{*}\right\| \leq \frac{Q-3|q| Q}{1+|q|}-H-\frac{b}{a_{|x| \leq H+Q}} \sup _{|\leq f(x)|} \mid
$$

then the solution $u(\psi)(t)$ through $\psi$ to $(2)$ satisfying $u(\psi)(t) \rightarrow$ $u^{*}(t)$ as $t \rightarrow \infty$, where $u^{*}(t)$ is a T-periodic solution of (2).

Remark 10. The sufficient conditions for the existence of periodic solutions in [15] are very complicated. For example, they need extra condition $Q>H, m<Q-H$ and

$$
\begin{gathered}
|q|<\frac{Q-H-m}{3 Q+H+m}, \\
\left\|\psi-u^{*}\right\| \leq \frac{Q-3|q| Q}{1+|q|}-H-\frac{m}{1+|q|},
\end{gathered}
$$

where $m=(b / a) \sup _{|x| \leq H+Q}|f(x)|$.

Theorem 11. If all conditions of Theorem 7 are satisfied, and the Lipschitz constant $L$ for $f$ corresponding to $(-\infty,+\infty)$ satisfies $1-3|q|-(b / a)(1+|q|) L>0$, then the T-periodic solution $u^{*}(t)$ of $(2)$ is stable.

Theorem 12. Suppose that $p$ is constant, the equation $p-a(1+$ $q) u=b(1-q) f(u)$ has only one solution $u^{*}, f \in C^{1}(\mathbb{R})$, and $f^{\prime}\left(u^{*}\right)=0$; then the equilibrium $u^{*}$ of (2) is exponentially asymptotically stable.

Example 13 (Lopes [9]). Consider the NFDE (3) which arises from a transmission line model, where $C_{0}>0, Z>0, r>$ $0,|k|<1, p \in C(\mathbb{R})$ and $f$ is a given nonlinear function. Let $\tilde{p}(t)=\left(1 / C_{0}\right) p(t), \tilde{a}=Z / C_{0}$, and $\tilde{f}(u)=\left(1 / C_{0}\right) f(u)$; then (3) can be rewritten as

$$
\begin{aligned}
u^{\prime}(t)-k u^{\prime}(t-r)= & \widetilde{p}(t)-\tilde{a} u(t)-\tilde{a} k u(t-r) \\
& -\widetilde{f}(u(t)-k u(t-r)) .
\end{aligned}
$$

Now, let $h(u(t), u(t-r))=\tilde{f}(u(t)-k u(t-r))$. It is not difficult to see that (43) is a special case of (1). Therefore, by Theorems $2-5$, we have the following.

Theorem 14. Suppose that $f \in C(\mathbb{R})$ and $p \in C_{T}$. If there exists a constant $H>0$ such that

$$
\|p\|_{0}<(1-3|k|) Z H-\sup _{|x| \leq(1+|k|) H}|f(x)|
$$

then (3) has a T-periodic solution.

Theorem 15. Let $H$ be as in Theorem 14. Assume that all conditions of Theorem 14 are satisfied. If $f$ satisfies the Lipschitz condition, $\sup _{|x| \leq(1+|k|) H}|f(x)| \leq Z H(1+|k|)$, and there exists $Q>0$ such that

$$
\begin{aligned}
& \left\|\psi-u^{*}\right\| \\
& \quad \leq \frac{Q-3|k| Q}{1+|k|}-H-\frac{1}{Z(1+|k|)} \sup _{|x| \leq(1+|k|)(H+Q)}|f(x)|,
\end{aligned}
$$

then the solution through $\psi$ of $(3) u(\psi)(t) \rightarrow u^{*}(t)$ as $t \rightarrow$ $\infty$, where $u^{*}(t)$ is a T-periodic solution of (3).

Theorem 16. If all conditions of Theorem 14 are satisfied, and the Lipschitz constant $L$ for $f$ corresponding to $(-\infty,+\infty)$ satisfies $1-3|k|-(L / Z)(1+|k|)>0$, then $T$-periodic solution $u^{*}(t)$ of $(3)$ is stable.

Theorem 17. Suppose that $p$ is constant, the equation $p-Z(1+$ $k) u=f(u-k u)$ has only one solution $u^{*}, f \in C^{1}(\mathbb{R})$, and $f^{\prime}\left(u^{*}-k u^{*}\right)=0$, then the equilibrium $u^{*}$ of (3) is exponentially asymptotically stable.

\section{Acknowledgment}

This work was partially supported by the National Natural Science Foundation of China nos. 11201411 and 11031002.

\section{References}

[1] R. K. Brayton, "Bifurcation of periodic solutions in a nonlinear difference-differential equations of neutral type," Quarterly of Applied Mathematics, vol. 24, pp. 215-224, 1966.

[2] R. K. Brayton, "Nonlinear oscillations in a distributed network," Quarterly of Applied Mathematics, vol. 24, pp. 289-301, 1967.

[3] R. K. Brayton and W. L. Miranker, "A stability theory for nonlinear mixed initial boundary value problems," Archive for Rational Mechanics and Analysis, vol. 17, pp. 358-376, 1964.

[4] R. K. Brayton and J. K. Moser, "A theory of nonlinear networks. I," Quarterly of Applied Mathematics, vol. 22, pp. 1-33, 1964.

[5] K. L. Cooke and D. W. Krumme, "Differential-difference equations and nonlinear initial-boundary value problems for linear hyperbolic partial differential equations," Journal of Mathematical Analysis and Applications, vol. 24, pp. 372-387, 1968.

[6] J. M. Ferreira, "On the stability of a distributed network," SIAM Journal on Mathematical Analysis, vol. 17, no. 1, pp. 38-45, 1986.

[7] W. Krawcewicz, J. Wu, and H. Xia, "Global Hopf bifurcation theory for condensing fields and neutral equations with applications to lossless transmission problems," The Canadian Applied Mathematics Quarterly, vol. 1, no. 2, pp. 167-220, 1993.

[8] O. Lopes, "Forced oscillations in nonlinear neutral differential equations," SIAM Journal on Applied Mathematics, vol. 29, pp. 196-207, 1975.

[9] O. Lopes, "Stability and forced oscillations," Journal of Mathematical Analysis and Applications, vol. 55, no. 3, pp. 686-698, 1976.

[10] S. Siqueira Ceron and O. Lopes, " $\alpha$-contractions and attractors for dissipative semilinear hyperbolic equations and systems," Annali di Matematica Pura ed Applicata. Serie Quarta, vol. 160, pp. 193-206, 1991. 
[11] S. Lu and W. Ge, "On the existence of periodic solutions for neutral functional differential equation," Nonlinear Analysis: Theory, Methods \& Applications, vol. 54, no. 7, pp. 1285-1306, 2003.

[12] W. L. Miranker, "Periodic solutions of the wave equation with a nonlinear interface condition," International Business Machines Corporation, vol. 5, pp. 2-24, 1961.

[13] M. Slemrod, "Nonexistence of oscillations in a nonlinear distributed network," Journal of Mathematical Analysis and Applications, vol. 36, pp. 22-40, 1971.

[14] J. J. Wei and S. G. Ruan, "Stability and global Hopf bifurcation for neutral differential equations," Acta Mathematica Sinica. Chinese Series, vol. 45, no. 1, pp. 93-104, 2002 (Chinese).

[15] L. Ding and Z. Li, "Periodicity and stability in neutral equations by Krasnoselskii's fixed point theorem," Nonlinear Analysis: Real World Applications, vol. 11, no. 3, pp. 1220-1228, 2010.

[16] T. A. Burton, "Perron-type stability theorems for neutral equations," Nonlinear Analysis: Theory, Methods \& Applications, vol. 55, no. 3, pp. 285-297, 2003.

[17] V. B. Kolmanovskiǔ and V. R. Nosov, Stability of functionaldifferential equations, vol. 180 of Mathematics in Science and Engineering, Academic Press, London, UK, 1986.

[18] Y. Kuang, Delay Differential Equations with Applications in Population Dynamics, vol. 191 of Mathematics in Science and Engineering, Academic Press, Boston, Mass, USA, 1993.

[19] T.-R. Ding and C. Li, Ordinary Differential Equations Tutorial, Higher Education Press, Bejing, China, 2004.

[20] J. K. Hale, "Functional differential equations," in Analytic Theory of Differential Equations, vol. 183 of Lecture Notes in Mathematics, p. 2, Springer, Berlin, Germany, 1971.

[21] D. X. Xia, Z. R. Wu, S. Z. Yan, and W. C. Shu, Real Variable Function and Functional Analysis, Higher Education Press, Beijing, China, 2nd edition, 2010.

[22] D. R. Smart, Fixed Point Theorems, Cambridge University Press, London, UK, 1974.

[23] V. G. Angelov, "Lossy transmission lines terminated by R-loads with exponential V-I characteristics," Nonlinear Analysis: Real World Applications, vol. 8, no. 2, pp. 579-589, 2007. 


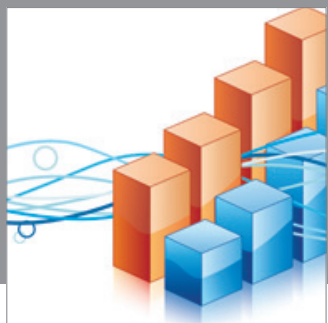

Advances in

Operations Research

mansans

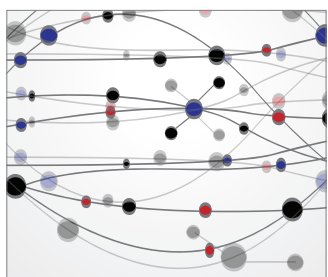

The Scientific World Journal
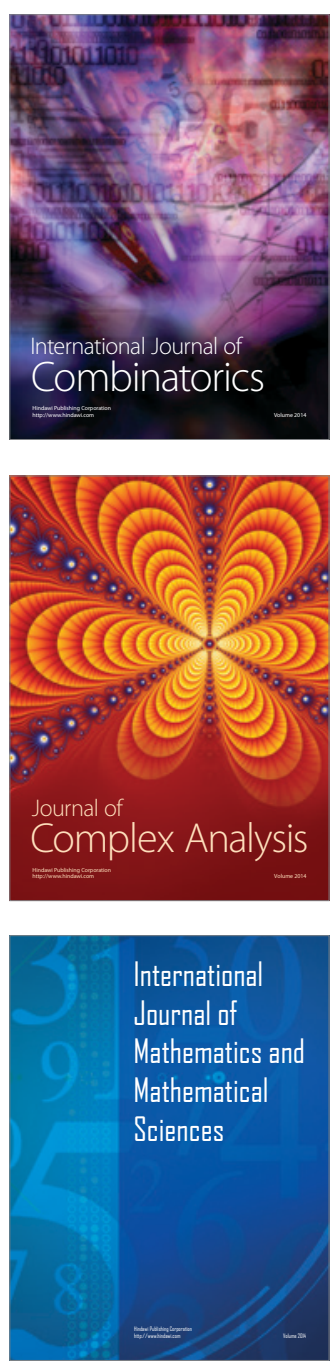
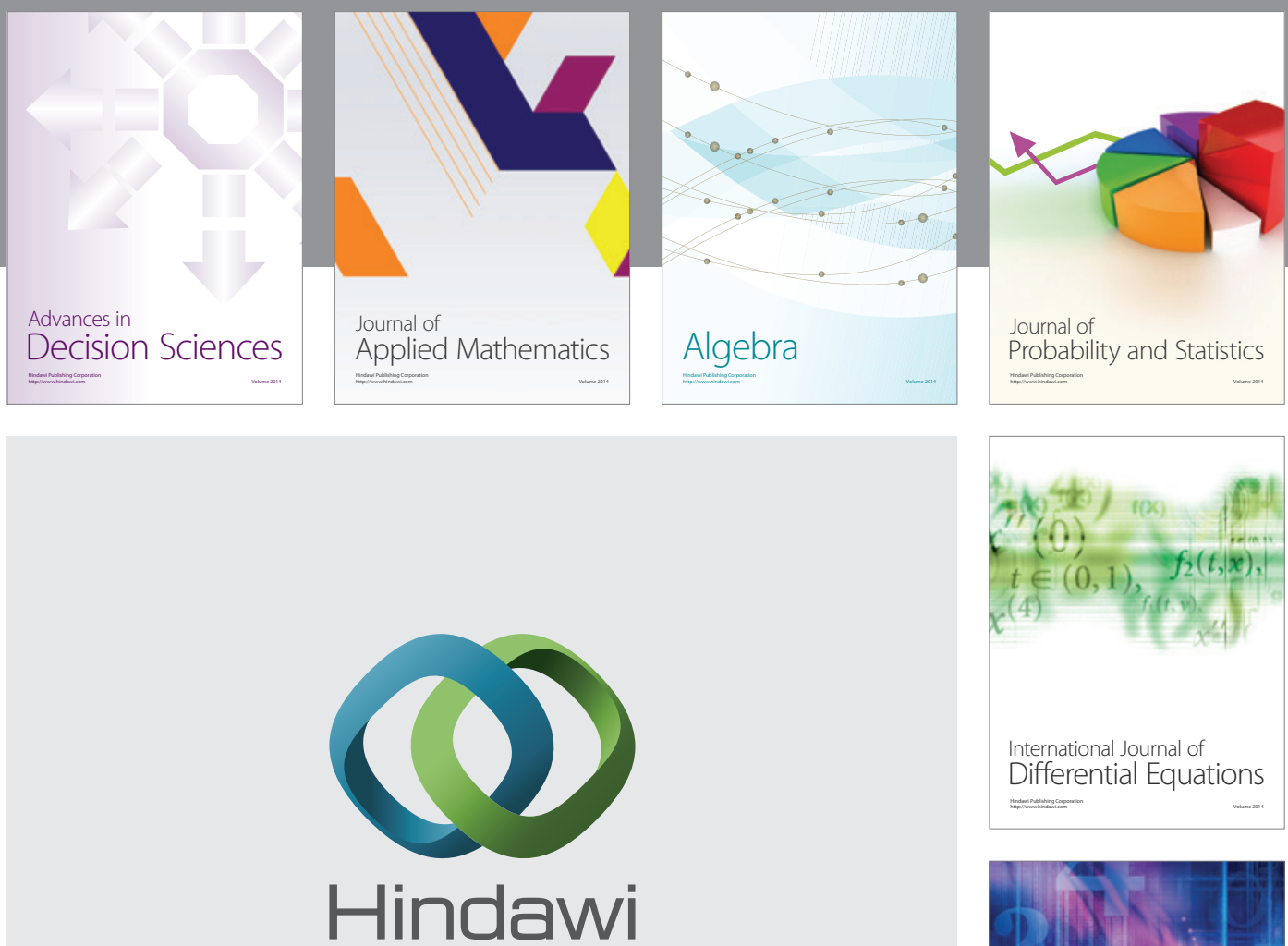

Submit your manuscripts at http://www.hindawi.com
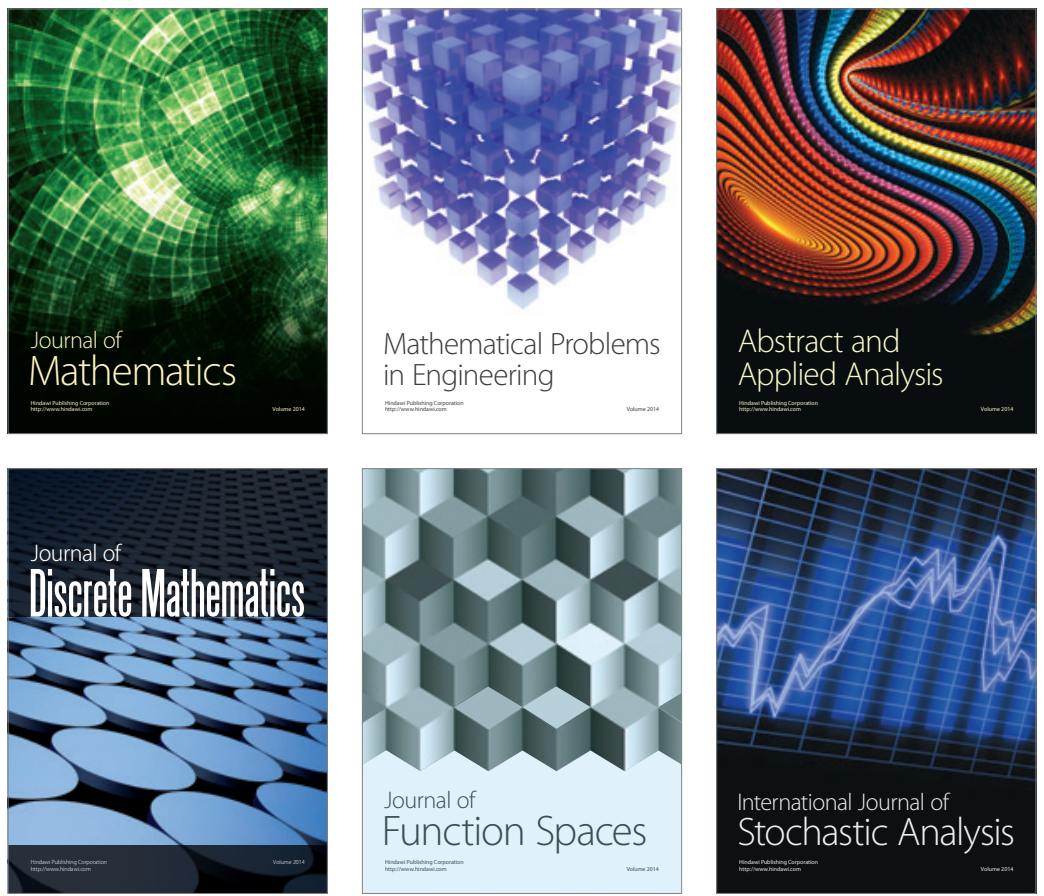

Journal of

Function Spaces

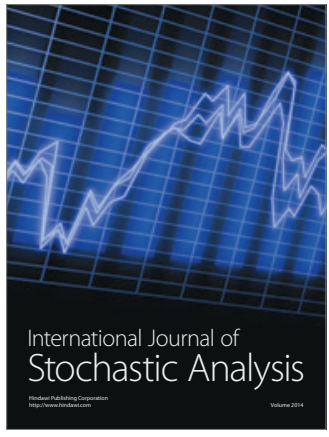

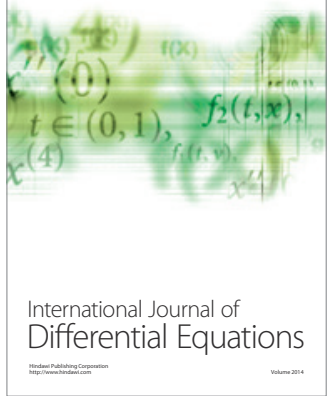
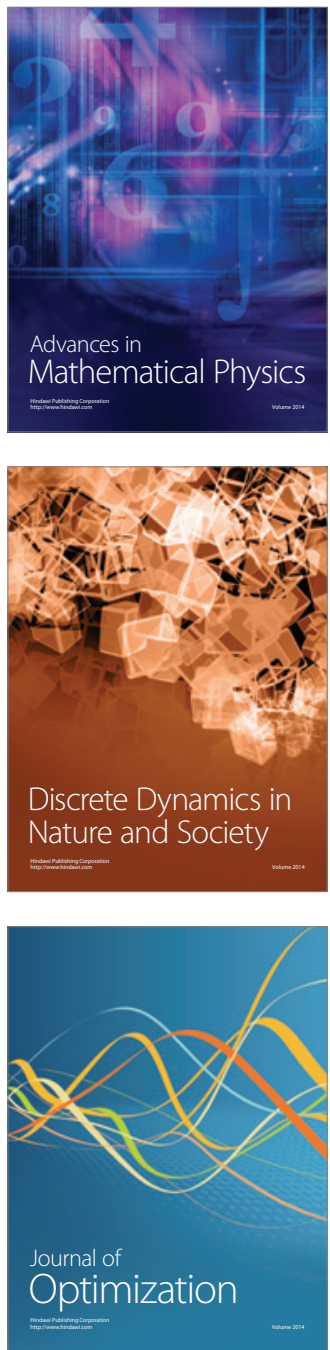